\title{
Zumbido no trabalhador exposto ao ruído
}

\author{
Tinnitus in noise-exposed workers
}

\author{
Sandra Regina Weber ${ }^{1}$, Eduardo Périco ${ }^{2}$
}

\begin{abstract}
RESUMO
Objetivo: Verificar as características, prevalência e repercussão do zumbido em trabalhadores expostos ao ruído. Métodos: Um total de 585 trabalhadores de uma indústria alimentícia foi questionado sobre zumbido. Aqueles que referiram zumbido por mais de cinco minutos e há mais de seis meses responderam à entrevista abordando histórico ocupacional e do zumbido, responderam ao questionário Tinnitus Handicap Inventory, e realizaram audiometria tonal. Foi realizada análise estatística dos dados. Resultados: Foi encontrada prevalência do zumbido de 7,2\% (n=42), tempo médio de exposição ao ruído de três anos e oito meses e tempo médio de percepção do zumbido de três anos e sete meses. Verificou-se predomínio da percepção intermitente (88\%), bilateral $(53,4 \%)$, com início progressivo $(66,7 \%)$. Um total de $50 \%$ não apresentou perda auditiva. Os sujeitos referiram que o zumbido interfere principalmente na concentração; estresse, silêncio e barulho foram os fatores de piora mais citados. Houve diferença entre o tempo de exposição ao ruído e o tempo de percepção do zumbido nos indivíduos sem perda auditiva. Conclusão: A ausência de perda auditiva em metade dos indivíduos e a correlação entre tempo de exposição ao ruído e tempo de percepção do zumbido, nestes indivíduos, apontam para um possível efeito do ruído não limitado ao sistema auditivo periférico e para a necessidade de inclusão do zumbido nos programas de conservação auditiva.
\end{abstract}

Descritores: Zumbido; Prevalência; Perda auditiva; Indústria alimentícia; Ruído ocupacional; Qualidade de vida; Saúde do trabalhador; Questionários

\section{INTRODUÇÃO}

O zumbido é definido como a percepção consciente de um som na ausência de fonte externa ${ }^{(1)}$. É um sintoma, não uma doença, e pode estar associado a múltiplas etiologias, inclusive à interação entre elas ${ }^{(2)}$.

O zumbido é considerado uma desordem fisiológica resultante de atividade neuronal anormal nas vias auditivos e erroneamente interpretada como som, nos centros corticais ${ }^{(3)}$. Concepções atuais sugerem que, nessa condição, há o envolvimento do sistema auditivo periférico e central, aferente e eferente e a interação com outros sistemas ${ }^{(3,4)}$.

Para alguns indivíduos, o zumbido é somente uma percepção auditiva, enquanto para outros, devido à ativação do sistema límbico e do sistema nervoso autônomo ${ }^{(5)}$, provoca impacto negativo e sofrimento ${ }^{(5,6)}$. Isso ocorre em aproximadamente $4 \%$ da população, com importante repercussão na qualidade de vida ${ }^{(1)}$.

Trabalho realizado no Programa de Pós-Graduação em Ambiente e Desenvolvimento, Centro Universitário Univates - Lajeado (RS), Brasil.

(1) Programa de Pós-graduação (Mestrado) em Ambiente e Desenvolvimento, Centro Universitário Univates - Lajeado (RS), Brasil.

(2) Programa de Pós-Graduação em Ambiente e Desenvolvimento, Centro Universitário Univates - Lajeado (RS), Brasil.

Endereço para correspondência: Sandra Regina Weber. R. Bento Gonçalves, 927/204, Centro, Lajeado (RS), Brasil, CEP: 95900-000. E-mail: sandra_seaf@yahoo.com.br

Recebido em: 9/8/2010; Aceito em: 23/11/2010
Estima-se que o zumbido ocorra em 10 a $15 \%$ da população geral, aumentando para 20 a $40 \%$ nos idosos ${ }^{(7)}$.

As queixas mais frequentes referem-se à interferência no sono, persistência do zumbido, dificuldade para entender a fala, desânimo, frustração ou depressão, incômodo, irritação, inabilidade para relaxar e dificuldade de concentração ou confusão $^{(8)}$.

No trabalhador exposto ao ruído, o zumbido tem sido associado à perda auditiva induzida pelo ruído (PAIR). A prevenção do desencadeamento ou agravamento da PAIR, conforme postulam os programas de conservação auditiva ${ }^{(9)}$, pode não ter eficácia para prevenir o zumbido ou para minimizar seu impacto na qualidade de vida do indivíduo.

O presente trabalho procurou estimar a ocorrência do zumbido nos trabalhadores expostos ao ruído; relacionar o zumbido com exposição ao ruído e com perda auditiva, avaliar a gravidade do zumbido na vida do trabalhador e identificar os fatores que melhoram ou pioram a percepção do zumbido.

\section{MÉTODOS}

O estudo foi desenvolvido em uma empresa do ramo de alimentação do município de Lajeado - RS. A empresa conta com 585 funcionários expostos a ruído, 372 homens e 213 mulheres e desenvolve programas de controle do ruído, prevenção e monitoramento de perda auditiva.

O trabalho foi aprovado pelo Comitê de Ética em Pesquisa 
do Centro Universitário Univates (COEP - projeto número 119/09) e o Termo de Consentimento Livre e Esclarecido, assinado por todos os participantes.

Os funcionários foram recrutados mediante comunicado escrito, divulgado em cada um dos 11 setores da empresa: bala diet, bala dura, bala mastigável, calda, chicle, empacotamento I, empacotamento II, extrusado, pirulito pelota, pirulito plano e salgado. O chefe de cada setor foi encarregado de listar os funcionários que apresentavam zumbido (som nos ouvidos ou na cabeça, como chiado, apito, buzina, grilo, chuva, motor, entre outros.) e que se disponibilizavam a participar da pesquisa. Considerando que zumbido espontâneo, com duração de poucos segundos ou minutos, é uma sensação praticamente universal $^{(6)}$, para este estudo, o zumbido foi definido como percepção de um som por mais de cinco minutos e há mais de seis meses ${ }^{(10)}$.

Os funcionários listados foram atendidos individualmente, pela pesquisadora, na própria empresa, durante o turno de trabalho. A empresa informou o número de funcionários e os níveis de pressão sonora de cada setor, de acordo com os registros do Programa de Prevenção de Riscos Ambientais (PPRA).

Foi realizada inspeção visual do meato acústico externo com uso de otoscópio, marca Heine®, modelo Mini Heine, de fibra ótica. Os trabalhadores com presença de cerume ou corpo estranho que obstruísse a passagem do som foram encaminhados ao médico otorrinolaringologista (ORL) para conduta pertinente. Trabalhadores com secreção no conduto auditivo externo, com perfuração da membrana timpânica ou com dor, foram excluídos da pesquisa.

Os funcionários responderam à entrevista ${ }^{(11)}$ abordando história e caracterização do zumbido, fatores de melhora, de piora e preocupação com o zumbido e interferência do zumbido em atividades diárias ${ }^{(11)}$. Foi realizada audiometria tonal em repouso auditivo de 14 horas, uso do audiômetro da marca Interacoustics ${ }^{\circledR}$, modelo AD 229, fones TDH-39, marca Telephonics ${ }^{\circledR}$, coxim modelo MX-41/AR, calibrado conforme as normas internacionais (ISO 8253); em cabina acústica da marca São Luiz $®$, modelo 1130. Foram avaliadas as frequências de 0.25 a $8 \mathrm{kHz}$, por via aérea. Ao ser encontrado limiar superior a $25 \mathrm{~dB}$, realizou-se também a pesquisa por via óssea, de 0.5 a $4 \mathrm{kHz}$. Como este procedimento é feito rotineiramente na empresa, considerou-se a última audiometria de cada funcionário, com data inferior a seis meses. Nos casos distintos, foi feita nova audiometria.

As audiometrias foram classificadas conforme critério da portaria 19, do Ministério do Trabalho. São considerados dentro dos limites aceitáveis, os casos cujos audiogramas apresentem limiares auditivos menores ou iguais a $25 \mathrm{~dB}$ (NA) em todas as frequências. Sugestivos de PAIR, os casos cujos audiogramas nas frequências de 3 e/ou 4 e/ou 6 kHz demonstrarem limiares auditivos superiores a $25 \mathrm{~dB}(\mathrm{NA})$, tanto no teste por via aérea quanto por via óssea, em um ou ambos os lados. São considerados não sugestivos de PAIR os casos cujos audiogramas não se enquadram nas descrições acima ${ }^{(12)}$.

Foi utilizado o questionário Tinnitus Handicap Inventory - $\mathrm{THI}^{(13)}$, traduzido e adaptado para a língua portuguesa ${ }^{(14)}$. O questionário é constituído por 25 perguntas, avaliando componente funcional: dificuldade de concentração ou leitura, dificuldade social, laboral e interferência na audição; componente afetivo: frustração, ansiedade, depressão e componente catastrófico: desespero e incapacidade do paciente acometido pelo zumbido. Para cada pergunta deve ser escolhida uma das respostas: sim, não ou às vezes. Estas respostas são tabuladas, considerando quatro pontos para a resposta sim, dois pontos para a resposta às vezes e nenhum ponto para a resposta não. Os pontos são somados a fim de mensurar a gravidade do zumbido: desprezível, leve, moderado, severo ou catastrófico.

\section{Análise estatística}

Foram realizadas análises descritivas dos dados encontrados na entrevista relacionados ao tempo de exposição ao ruído, tempo de percepção e características do zumbido, história familiar, perda auditiva, doenças e sintomas associados.

A análise estatística foi realizada a partir da correlação de Spearman, do teste de $\chi^{2}$, teste $t$ e teste G, considerando significativo $\mathrm{p}<0,05$.

A correlação de Spearman foi utilizada para verificar a associação entre o percentual de indivíduos com zumbido, encontrados em cada setor e o nível de ruído; gravidade do zumbido (medida pelo THI) e o tempo de percepção do zumbido; correlação entre o tempo de exposição ao ruído e o tempo de percepção do zumbido nos indivíduos com e sem perda auditiva.

O teste de $\chi^{2}$ foi utilizado para testar a plenitude auricular, tontura e sensibilidade auditiva em relação a indivíduos com e sem perda auditiva.

O teste $\mathrm{t}$ foi usado para verificar a relação entre a média do tempo de exposição ao ruído nos indivíduos com e sem perda auditiva e a relação entre a gravidade do zumbido (THI) nos indivíduos com e sem perda auditiva.

$\mathrm{O}$ teste $\mathrm{G}$ foi utilizado para verificar se o barulho como fator de piora do zumbido e a sensibilidade aos sons estão relacionados com o nível de ruído do local de trabalho.

\section{RESULTADOS}

A coleta de dados ocorreu no período de agosto a dezembro de 2009. Dos 585 funcionários da empresa, inicialmente, 62 funcionários $(10,6 \%)$ referiram percepção de zumbido. Durante a avaliação fonoaudiológica foi constatado que dois funcionários apresentavam excesso de cera no meato acústico externo, foram encaminhados ao otorrinolaringologista e ocorreu supressão do zumbido após a remoção; um funcionário apresentava surdez profunda, não reabilitada; dois funcionários apresentavam doença ativa de orelha; três percebiam o zumbido há menos de seis meses e/ou menos de cinco minutos; quatro foram demitidos e oito não entenderam a entrevista inicial, supondo tratar-se de um estudo sobre barulho ao qual estão expostos. Desta forma, foram excluídos 20 funcionários.

A amostra final foi composta por 42 funcionários, correspondendo a $7,2 \%$ da população total $(\mathrm{n}=585), 25$ do gênero masculino (59,5\%) e 17 do gênero feminino (40,5\%). A faixa etária variou de 20 a 60 anos, com predomínio entre 30 e 40 anos $(33,3 \%)$ e a média da idade de 35 anos e 6 meses. No 
grupo das mulheres, a idade variou de 21 a 60 anos, com média de 35 anos e 5 meses. No grupo de homens a idade variou de 20 a 49 anos, com média de 35 anos.

O nível de ruído nos diferentes setores da empresa variou de 81,2 dB (A) a 95,5 dB (A). Pela correlação de Spearman, não houve associação significativa entre o nível de ruído do setor e o percentual de indivíduos com zumbido ( $r s=0,21$; $\mathrm{p}=0,51)$. Em outras palavras, o setor cujo nível de ruído é mais intenso não apresenta o maior percentual de indivíduos com zumbido (Figura 1).

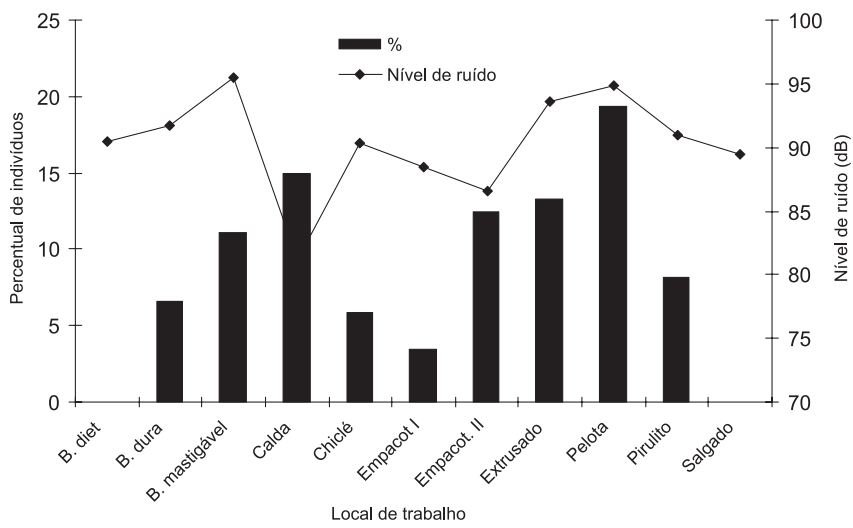

Legenda: B. diet = bala diet; B. dura = bala dura; B. mastigável = bala mastigável; Empacot. I = empacotamento I; Empacot.II = empacotamento II

Figura 1. Percentual de indivíduos que apresentam zumbido, distribuídos por local de trabalho e nível de ruído em cada local

O tempo de exposição ao ruído variou de um a 30 anos. A maioria dos indivíduos $(57,6 \%)$ tem até dez anos de exposição ao ruído, com média de três anos e oito meses (Tabela 1). A aplicação do teste $t$ não revelou diferença significativa entre a média de tempo de exposição ao ruído nos indivíduos sem perda auditiva $(X=133,19)$ e nos indivíduos com perda auditiva $(\mathrm{X}=146,28) ;(\mathrm{t}=-0,42 ; \mathrm{gl}=40 ; \mathrm{p}=0,67)$.

Tabela 1. Frequência de indivíduos por tempo de exposição ao ruído

\begin{tabular}{ccc}
\hline Tempo (anos) & $\mathrm{N}^{\circ}$ indivíduos & Frequência \\
\hline $1+5$ & 10 & 23,8 \\
$5+10$ & 10 & 23,8 \\
$10+15$ & 5 & 11,9 \\
$15+20$ & 7 & 16,6 \\
$20+25$ & 9 & 21,4 \\
$25+30$ & 1 & 2,4 \\
\hline
\end{tabular}

Os resultados da audiometria tonal mostraram 21 funcionários (50\%) com limiares audiométricos nos padrões de normalidade. Os demais indivíduos apresentaram limiares acima de $25 \mathrm{~dB}$ (NA), com predomínio da configuração audiométrica compatível com PAIR (14 funcionários, 33\%).

Foram calculadas as medianas dos limiares audiométricos para a orelha direita e para a orelha esquerda. Observa-se que na configuração audiométrica compatível com PAIR os limiares são simétricos com alterações que não excedem $35 \mathrm{~dB}$ (NA) e limitados às frequências de 3 e 4 kHz (Figuras 2 e 3 ).

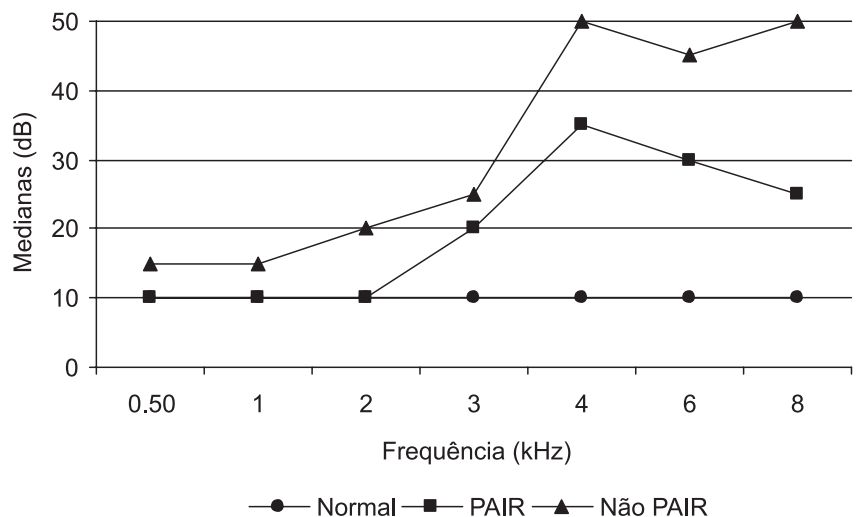

Figura 2. Medianas para os limiares audiométricos, para a orelha direita, dos grupos com limiares audiométricos normais, sugestivos de PAIR e não sugestivos de PAIR

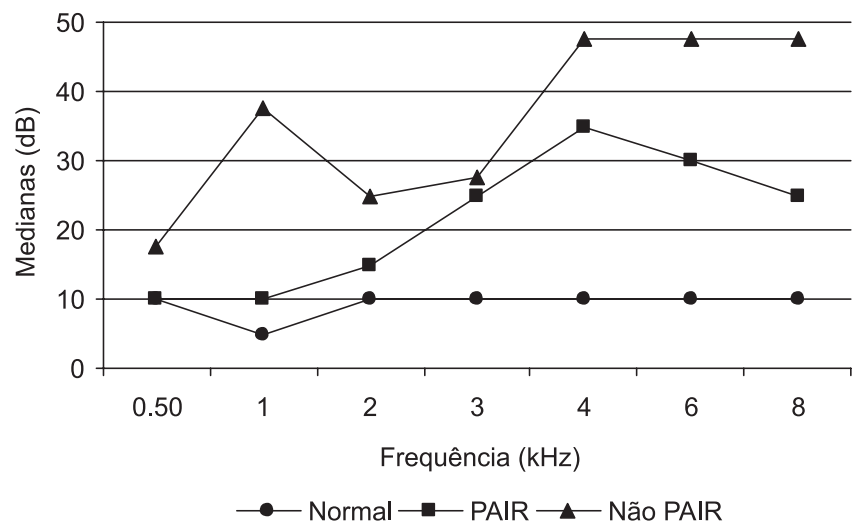

Figura 3. Medianas para os limiares audiométricos, para a orelha esquerda, dos grupos com limiares audiométricos normais, sugestivos de PAIR e não sugestivos de PAIR

Também não foi encontrada diferença entre indivíduos com e sem perda auditiva, quando considerados, pelo teste de $\chi^{2}$, as variáveis plenitude auricular $\left(\chi^{2}=0,11 ; \mathrm{gl}=1 ; \mathrm{p}=0,73\right)$; sensibilidade aos sons $\left(\chi^{2}=0,0\right)$ e tontura $\left(\chi^{2}=0,38 ; \mathrm{gl}=1\right.$; $\mathrm{p}=0,53)$.

O tempo de percepção do zumbido variou de seis meses a 23 anos, a maioria dos indivíduos $(73,8 \%)$ percebe o zumbido há menos de cinco anos, com média de três anos e sete meses. Para oito indivíduos (19\%) a percepção do sintoma ocorre há menos de um ano. Para a maioria, o zumbido é bilateral, percebido como som único, intermitente, de início progressivo (Tabela 2).

Considerando o coeficiente de correlação de Spearman, não foi encontrada associação significativa entre tempo de exposição ao ruído e tempo de percepção $\mathrm{d}$ zumbido nos indivíduos com perda auditiva ( $r s=0,268 ; p=0,239)$. Entretanto, nos indivíduos que não apresentam perda auditiva, foi encontrada correlação significativa ( $r s=0,552 ; p=0,009)$, indicando, neste grupo, haver relação entre o tempo de exposição ao ruído e o tempo de percepção do zumbido.

O questionário THI mostrou que 17 indivíduos (40,5\%) encontram-se no nível 1 ou desprezível, mas nenhum indivíduo encontra-se no nível catastrófico. O teste t não mostrou diferença significativa entre a gravidade do zumbido (THI) nos indivíduos com perda auditiva $(X=2,09)$ e nos indivíduos sem perda auditiva $(X=1,90)(t=-0,591, \mathrm{gl}=40$ e $\mathrm{p}=0,557)$. 
Tabela 2. Caracterização do zumbido

\begin{tabular}{|c|c|c|c|c|c|c|c|c|c|c|c|c|c|c|}
\hline \multicolumn{3}{|c|}{ Tempo (anos) } & \multicolumn{3}{|c|}{ Localização } & \multicolumn{3}{|c|}{ Tiрo } & \multicolumn{3}{|c|}{ Forma de início } & \multicolumn{3}{|c|}{ Percepção } \\
\hline & $\mathrm{n}$ & $\%$ & & $\mathrm{n}$ & $\%$ & & $n$ & $\%$ & & $n$ & $\%$ & & $n$ & $\%$ \\
\hline $0,5+5$ & 31 & 73,8 & $\mathrm{D}$ & 5 & 11,9 & Único & 36 & 85,7 & Progressivo & 27 & 66,7 & Intermitente & 37 & 88 \\
\hline 5 卜 10 & 6 & 14,2 & $E$ & 9 & 21,4 & Múltiplo & 4 & 9,5 & Súbito & 15 & 33,3 & Constante & 5 & 12 \\
\hline 10 아 15 & 4 & 9,5 & $\mathrm{D}=\mathrm{E}$ & 8 & 19,1 & Pulsátil & 2 & 4,8 & & & & & & \\
\hline $15+20$ & 0 & 0 & $\mathrm{D}>\mathrm{E}$ & 7 & 16,6 & Clique & 0 & 0 & & & & & & \\
\hline \multirow[t]{2}{*}{$20+25$} & 1 & 2,3 & $E>D$ & 6 & 14,3 & & & & & & & & & \\
\hline & & & Cabeça & 7 & 16,6 & & & & & & & & & \\
\hline
\end{tabular}

Legenda: $\mathrm{D}$ = orelha direita; $\mathrm{E}=$ orelha esquerda

Também foi testada a correlação entre a gravidade do zumbido (THI) e o tempo de percepção do zumbido. Não foi encontrada associação significativa ( $r s=-0,051 ; p=0,744)$, indicando não haver relação entre as variáveis.

Com relação às doenças associadas, $64,2 \%$ não referiram nenhuma doença; $14,2 \%$ referiram depressão; $7,2 \%$ doença metabólica; 7,2\% doença do sistema músculo- esquelético; $4,2 \%$ doença cardiovascular e 2,4\% doença neurológica.

Os dados relacionados ao histórico familiar indicam que 54,7\% apresentam algum familiar com zumbido, perda auditiva ou doença metabólica. Com relação ao consumo de doces, 54,2\% acreditam abusar no consumo e 16,7\% abusam do consumo de cafeína.

O estresse, o silêncio e o barulho foram os fatores de piora mais citados. Para 43\%, o uso de som ambiental melhora o zumbido. Para $34 \%$ dos indivíduos, o zumbido interfere principalmente na concentração e $28 \%$ preocupam-se com surdez (Tabela 3).

Para avaliar se o barulho como fator de piora do zumbido está relacionado com o nível de ruído do local de trabalho, foi utilizado o teste G. Não houve diferença significativa entre nível de ruído e piora com barulho ( $\mathrm{p}=0,809)$, indicando não haver relação entre ambos.

Também foi utilizado o teste G para avaliar se a queixa de sensibilidade aos sons está relacionada ao nível de ruído do local de trabalho. Não houve relação de significância ( $\mathrm{p}=0,754)$.

\section{DISCUSSÃO}

O zumbido é um sintoma multifacetado e complexo que pode comprometer dramaticamente a qualidade de vida do indivíduo ${ }^{(2)}$. A incidência em nível mundial deverá crescer, considerando-se o aumento da idade da população, a crescente exposição ao ruído, o estilo de vida (hábitos alimentares, sobrecarga de trabalho, estresse), a ansiedade e a depressão.

As pesquisas com populações expostas ao ruído mostram grande variabilidade na prevalência do zumbido ${ }^{(15-19)}$ que pode ser atribuída aos métodos de pesquisa, à subjetividade do sintoma e à definição de zumbido.

A amostra constituiu-se de 42 indivíduos, com mais homens do que mulheres. Porém, analisando o total de homens e mulheres na empresa, encontrou-se maior percentual de mulheres do que de homens. Segundo a literatura, a prevalência do zumbido é maior em homens e aumenta com a ida$\mathrm{de}^{(6)}$. Considerando que a faixa etária média (35 anos e cinco meses) encontrada para as mulheres foi maior, esta poderia ser a justificativa para o maior percentual de mulheres com zumbido neste estudo.

Foi encontrada média de tempo de exposição ao ruído de três anos e oito meses e média de tempo de percepção do zumbido de três anos e sete meses. Segundo outros autores ${ }^{(15)}$, entre o início da exposição ao ruído ocupacional e o início do zumbido de origem ocupacional, em geral, há um intervalo

Tabela 3. THI, fatores de piora, fatores de melhora, interferência e preocupação

\begin{tabular}{|c|c|c|c|c|c|c|c|c|c|c|c|c|c|c|}
\hline \multicolumn{3}{|c|}{$\mathrm{THI}$} & \multicolumn{3}{|c|}{ Fatores de piora } & \multicolumn{3}{|c|}{ Fatores de melhora } & \multicolumn{3}{|c|}{ Interferência } & \multicolumn{3}{|c|}{ Preocupação } \\
\hline & $\mathrm{n}$ & $\%$ & & $\mathrm{n}$ & $\%$ & & $\mathrm{n}$ & $\%$ & & $\mathrm{n}$ & $\%$ & & $\mathrm{n}$ & $\%$ \\
\hline Ligeiro & 17 & 40 & Manhã & 5 & 12,2 & Não & 21 & 50 & Não & 22 & 52,4 & Não & 23 & 55 \\
\hline Leve & 13 & 31 & Noite & 16 & 39 & Som amb. & 18 & 43 & Sono & 8 & 19,5 & Surdez & 12 & 29 \\
\hline Mod. & 7 & 17 & Álcool & 2 & 4,87 & Medicação & 0 & 0 & Conc. & 14 & 34,1 & D. grave & 2 & 4,8 \\
\hline Sev. & 5 & 12 & Cigarro & 0 & 0 & Exercício & 1 & 2.3 & Emoc. & 7 & 17,1 & Piorar & 3 & 7,1 \\
\hline \multirow[t]{6}{*}{ Catas. } & 0 & 0 & Exercício & 6 & 14,6 & Aper. ore. & 1 & 2.3 & Social & 4 & 9,75 & Enlo. & 1 & 2,4 \\
\hline & & & Barulho & 16 & 39 & Afas. ruído & 1 & 2.3 & & & & Desc. & 1 & 2,4 \\
\hline & & & Silêncio & 18 & 42,9 & & & & & & & & & \\
\hline & & & Estresse & 25 & 61 & & & & & & & & & \\
\hline & & & Soneca dia & 1 & 2,43 & & & & & & & & & \\
\hline & & & Jejum & 4 & 9,75 & & & & & & & & & \\
\hline
\end{tabular}

Legenda: Mod. = moderado; Sev. = severo; Catas. = catastrófico; Aper. ore. $=$ apertar orelha; Afas. ruído = afastar do ruído; Conc.$=$ concentração; Emoc.$=$ emocional; D. grave = doença grave; Enlo. = enlouquecer; Desc. $=$ não saber o que é o som; Som amb. = som ambiental 
longo, de muitos anos; diferente de um zumbido provocado por trauma acústico ou por exposição à música excessivamente amplificada. Ou seja, para os autores, é incomum a relação direta entre o início de exposição a ruído ocupacional e o início do zumbido.

A literatura concorda com a estreita associação entre zumbido e perda auditiva, considerando que a maioria dos indivíduos com zumbido, em torno de $85 \%$, tem algum grau de perda auditiva ${ }^{(1)}$ e somente oito a $10 \%$ dos indivíduos apresentam audiometria normal ${ }^{(20)}$. Este estudo mostrou que $50 \%$ dos trabalhadores com zumbido não apresentaram perda auditiva, diferindo de outro ${ }^{(19)}$, em que $71 \%$ dos trabalhadores apresentaram limiares audiométricos inferiores a $25 \mathrm{~dB}$ NA nas frequências de 0,25 a $8 \mathrm{kHz}$.

Deve-se considerar que uma lesão difusa, de até $30 \%$ nas células ciliadas externas (CCEs), não teria impacto significativo nos limiares audiométricos, seria uma lesão sem representação na audiometria convencional ${ }^{(3)}$. Além disso, os efeitos do ruído no sistema auditivo não estão limitados apenas à injúria coclear; a exposição ao ruído aumenta a concentração de cálcio citoplasmático nas CCEs, aumenta a liberação de glutamato (neurotransmissor excitatório da via auditiva) e aumenta a atividade neural das fibras aferentes, podendo levar ao zumbido ${ }^{(21)}$.

Estudos com animais mostraram complexas modificações no sistema auditivo central, no colículo inferior ${ }^{(22)}$, no corpo geniculado medial e no córtex auditivo primário ${ }^{(23)}$. Há evidências de que a exposição a som intenso leva à hiperatividade do núcleo coclear dorsal (primeiro núcleo da via auditiva) ${ }^{(24)}$. Esta hiperatividade é contínua e gradual, desencadeando mudanças em toda a via tonotópica mesmo depois de cessada a exposição ao som, podendo estar relacionada ao zumbido.

A ocorrência de PAIR foi constatada em 33,3\% dos indivíduos e as medianas encontradas neste estudo, assim como em outro similar ${ }^{(19)}$, apresentaram alterações mínimas, com limiares em torno de $30 \mathrm{~dB}$ (NA), limitadas a 3, 4 e $6 \mathrm{kHz}$ (Figuras 2 e 3). A desvantagem provocada pelo zumbido pode ser muito maior do que uma pequena perda auditiva nas frequências agudas, especialmente para indivíduos jovens ${ }^{(15)}$.

A multiplicidade etiológica (causas metabólicas, odontológicas, farmacológicas, cardiovasculares, psicológicas, neurológicas, dentre outras) e a possibilidade de interação entre as diversas causas ${ }^{(2)}$ dificultam a tarefa de determinar a causa do zumbido. $\mathrm{O}$ indivíduo pode ser portador de uma doença que cause zumbido sem que essa relação causal tenha sido determinada ${ }^{(25)}$. Além disso, um fator etiológico pode não ser suficiente para desencadear o zumbido, mas sim vários fatores atuando sinergicamente ${ }^{(2,25)}$.

Ao avaliar a interferência do zumbido nas atividades diárias de pacientes do Ambulatório de Zumbido, outros autores ${ }^{(26)}$, encontraram interferência no sono como a mais referida, seguida da capacidade de concentração, equilíbrio emocional e restrição da atividade social. Neste estudo, a capacidade de concentração foi a interferência mais referida, seguida do sono, equilíbrio emocional e atividade social. Trata-se de populações distintas; na primeira, pacientes de um centro de referência na avaliação e tratamento do zumbido e na segunda, trabalhadores que foram avaliados em seu local de trabalho e cujos percentuais encontrados interrogam sobre a necessidade de alguma intervenção.

O início do zumbido foi apontado como progressivo para a maioria dos indivíduos, e com percepção há menos de cinco anos. Destes, $19 \%$ têm percepção precoce do zumbido, ou seja, há menos de um ano. Achados muito semelhantes aos de outro estudo ${ }^{(19)}$ que também encontrou $19 \%$ dos indivíduos com percepção do zumbido há menos de um ano e, 67\% com percepção entre um e cinco anos.

A localização do zumbido é predominantemente bilateral, seguida da orelha esquerda, coincidindo com outros acha$\operatorname{dos}^{(15,16,19)}$.

A exposição a som ambiental foi o fator de melhora mais apontado pelos indivíduos. Protocolos de reabilitação como Tinnitus Retraining Therapy ${ }^{(27)}$ preconizam o uso de enriquecimento sonoro. De uma forma geral, todos os pacientes são orientados a evitar o silêncio, a fim de diminuir a atenção e a percepção do zumbido, reduzir sua intensidade e oferecer um som menos intrusivo. Empiricamente, $42,9 \%$ dos indivíduos já haviam percebido este benefício. Outros $50 \%$ não apontaram nenhum fator de melhora.

$\mathrm{O}$ estresse e o barulho, assim como o silêncio, foram os fatores mais citados para piora do zumbido. Para alguns pacientes o zumbido piora após exposição ao ruído. Esta diferença carece de entendimento e deve ser considerada numa abordagem terapêutica ${ }^{(8)}$. Considerando que a população estudada faz uso regular de protetor auricular e trabalha exposta ao ruído, analisamos se os indivíduos que fizeram esta referência encontram-se nos setores de ruído mais intenso. $\mathrm{O}$ teste $\mathrm{G}$ não mostrou diferença significativa.

Outros autores $^{(28)}$ realizaram a identificação de indivíduos com zumbido em subgrupos, a partir da análise de aglomerados. Encontraram inicialmente quatro subgrupos: constante incômodo com o zumbido, piora do zumbido no ruído, zumbido não influenciado pelo sistema somatossensorial, piora do zumbido no silêncio. Incluir o indivíduo com zumbido em uma destas quatro categorias pode ser uma importante ferramenta para otimizar a abordagem terapêutica e planejar ações profiláticas.

O estresse foi apontado como fator de piora do zumbido. Vários fatores cotidianos estão relacionados ao estresse. A própria exposição ao ruído e às suas características desagradáveis pode estar relacionada ao estresse ${ }^{(29)}$. O ruído é um agente de estresse psicossocial, que ativa o sistema endócrino e o sistema nervoso autônomo (simpático). Os efeitos da exposição não ocorrem somente diante de níveis de ruído intenso, mas também, em ambientes de ruído pouco intenso, quando há necessidade, por exemplo, de concentração ${ }^{(30)}$. Conforme o modelo neurofisiológico do zumbido ${ }^{(27)}$, ele ativa outros sistemas, como o sistema límbico e o sistema nervoso autônomo, levando ao incômodo e ao estresse. Uma vez ativado este mecanismo, o zumbido aumenta o estresse e, de maneira recíproca, o estresse aumenta o zumbido.

O zumbido inicia-se como uma percepção auditiva e, a persistência desta percepção, frequentemente, está associada a desordens de atenção e a problemas emocionais. O indivíduo passa a focar sua atenção no zumbido, desenvolvendo problemas de concentração. A percepção continuada do zumbido 
leva ao incômodo, frustração, medo, ansiedade e depressão. A associação de dificuldade de atenção e desordens emocionais culminaria com distúrbios do sono e um impacto ainda maior na qualidade de vida do indivíduo. Desta forma, os três componentes básicos do zumbido seriam: auditivo, atencional e emocional $^{(4)}$.

Considerando-se que foi encontrada perda auditiva em $50 \%$ da amostra estudada, foram realizadas análises a fim de investigar diferenças entre os indivíduos com perda auditiva e sem perda auditiva. Não foram encontradas diferenças significativas para média do tempo de exposição ao ruído, sensação de plenitude auricular, tontura, sensibilidade auditiva e severidade do zumbido.

Esta homogeneidade não se confirmou para a questão tempo de exposição ao ruído e tempo de percepção do zumbido. Nos indivíduos sem perda auditiva, encontrou-se diferença significativa entre tempo de exposição ao ruído e tempo de percepção do zumbido, indicando haver relação entre as variáveis. Deve-se considerar, por um lado, autores que sugerem haver um intervalo longo entre o início da exposição ao ruído ocupacional e o início do zumbido de origem ocupacional ${ }^{(15)}$.
Por outro lado, há que se valorizar o fato de que os efeitos do ruído podem ser imediatos à exposição, acarretando mudanças no sistema auditivo central, progressivas e gradativas, que podem estar relacionadas ao zumbido ${ }^{(24)}$. Neste estudo, encontramos uma correlação entre tempo de exposição ao ruído e tempo de percepção do zumbido, nos indivíduos sem perda auditiva, apontando para um possível efeito do ruído não limitado ao sistema auditivo periférico.

\section{CONCLUSÃO}

A ausência de perda auditiva em metade dos indivíduos estudados e a correlação entre tempo de exposição ao ruído e tempo de percepção do zumbido nestes indivíduos, aponta para um possível efeito do ruído não limitado ao sistema auditivo periférico. A similaridade entre os indivíduos com e sem perda auditiva quanto a diversas variáveis testadas (sensação de plenitude auricular, tontura, sensibilidade auditiva, média do tempo de exposição ao ruído e severidade do zumbido) indica a necessidade de incluir o tema zumbido nos programas de conservação auditiva.

\begin{abstract}
Purpose: To verify the characteristics, prevalence, and repercussion of tinnitus in noise-exposed workers. Methods: A total of 585 workers from a food industry were questioned about tinnitus. Individuals that reported tinnitus with duration of more than five minutes and for more than six months answered an interview regarding occupational and tinnitus histories, answered to the Tinnitus Handicap Inventory questionnaire, and carried out a pure tone audiometry. Data were statistically analyzed. Results: Tinnitus prevalence was $7.2 \%(\mathrm{n}=42)$, the average noise exposure time was three years and eight months, and the average tinnitus perception time was three years and seven months. There was a predominance of intermittent (88\%) and bilateral (53.4\%) perception, with progressive onset $(66.7 \%)$. A total of $50 \%$ didn't present hearing loss. The subjects reported that tinnitus interferes mainly in concentration; stress, silence and noise were the worsening factors most frequently mentioned. There was a difference between noise exposure time and tinnitus perception time in individuals without hearing loss. Conclusion: The absence of hearing loss in half the individuals and the correlation between noise exposure time and tinnitus perception time, in these subjects, suggest a possible effect of noise not restrained to the peripheral auditory system, and the need to include tinnitus in hearing conservation programs.
\end{abstract}

Keywords: Tinnitus; Prevalence; Hearing loss; Food industry; Noise, Occupational; Quality of life; Occupational health; Questionnaires

\title{
REFERÊNCIAS
}

1. Mc Fadden D. Tinnitus: facts, theories and treatments. Washington (DC): National Academy Press; 1982.p.1-150.

2. Sanchez TG e Ferrari GM. O que é zumbido? In: Samelli AG, organizador. Zumbido, avaliação, diagnóstico e reabilitação: abordagens atuais. São Paulo: Lovise; 2004. p.17-22.

3. Jastreboff PJ. Phantom auditory perception (tinnitus): mechanisms of generation and perception. Neurosci Res. 1990;8(4):221-54.

4. Kaltenbach JA. The dorsal cochlear nucleus as a participant in the auditory, attentional and emotional components of tinnitus. Hear Res. 2006;216-217:224-34.

5. Jastreboff PJ, Hazell JW. A neurophysiological approach to tinnitus: clinical implications. Br J Audiol. 1993;27(1):7-17.

6. Dobie RA. Overview: suffering from tinnitus. In: Snow JB. Tinnitus: theory and management. Lewiston (NY): BC Decker; 2004. p. 1-7.

7. Axelsson A, Ringdahl A. Tinnitus: a study of its prevalence and characteristics. Br J Audiol. 1989;23(1):53-62.

8. Tyler RS, Baker LJ. Difficulties experienced by tinnitus sufferers. J Speech Hear Disord. 1983;48(2):150-4.

9. Verbeek JH, Kateman E, Morata TC, Dreschler W, Sorgdrager B. Interventions to prevent occupational noise induced hearing loss. Cochrane Database Syst Rev. 2009;(3):CD006396 .

10. Heller AJ. Classification and epidemiology of tinnitus. Otolaryngol Clin North Am. 2003;36(2):239-48.

11. VI Curso Avançado de Zumbido do Hospital das Clínicas da Faculdade de Medicina de São Paulo; 2008 Dez 4-5; São Paulo.

12. Brasil. Ministério do Trabalho. Portaria n. 19 GM/SSSTb, de 9 de abril de 1998. Estabelece diretrizes e parâmetros mínimos para avaliação e acompanhamento da audição em trabalhadores expostos a níveis de pressão sonora elevados. Diário Oficial da República Federativa do Brasil, Brasília (DF); 1998 Abr 22. 
13. Newman CW, Jacobson GP, Spitzer JB. Development of the tinnitus handicap inventory. Arch Otolaryngol Head Neck Surg. 1996;122(2):143-8.

14. Ferreira PE, Cunha F, Onishi ET, Branco-Barreiro FC, Ganança FF. Tinnitus handicap inventory: adaptação cultural para o português brasileiro. Pró-Fono. 2005;17(3):303-10.

15. Axelsson A, Prasher D. Tinnitus induced by occupacional and leisure noise. Noise Health. 2000;2(8):47-54.

16. Possani LN. Estudo da prevalência e das características do zumbido em trabalhadores expostos ao ruído ocupacional [dissertação]. Porto Alegre: Universidade Federal do Rio Grande do Sul; 2006.

17. Dias A, Cordeiro R, Corrente JE, Gonçalves CG. Associação entre perda auditiva induzida pelo ruído e zumbidos. Cad Saúde Pública. 2006;22(1):63-8.

18. Rubak T, Kock S, Koefoed-Nielsen B, Lund SP, Bonde JP, Kolstad HA. The risk of tinnitus following occupacional noise exposure in workers with hearing loss or normal hearing. Int J Audiol. 2008;47(3):109-14.

19. Steinmetz LG, Zeigelboim BS, Lacerda AB, Morata TC, Marques JM. Características do zumbido em trabalhadores expostos a ruído. Rev Bras Otorrinolaringol. 2009;75(1):7-14.

20. Barnea G, Attias J, Gold S, Shahar A. Tinnitus with normal hearing sensitivity: extended high-frequency audiometry and auditory nerve brain-stem-evoked responses. Audiology. 1990;29(1):36-45.

21. Eggermont JJ. On the pathophysiology of tinnitus; a review and a peripherical model. Hear Res. 1990;48(1-2):111-23.

22. Basta D, Ernest A. Noise-induced changes of neuronal spontaneous activity in mice inferior colliculus brain slice. Neurosci Lett. 2004;368(3):297-302.
23. Basta D, Tzschentke B, Ernest A. Noise-induced cell death in the mouse medial geniculate body and primary auditory cortex. Neurosci Lett. 2005;381(1-2):199-204.

24. Kaltenbach JA, Zhang J, Afman CE. Plasticity of spontaneous neural activity in the dorsal cochlear after intense sound exposure. Hear Res. 2000;147(1-2):282-92.

25. Levine RA. Somatic tinnitus. In: Snow JB. Tinnitus: theory and management. Lewiston (NY): BC Decker; 2004. p.108-24.

26. Sanchez TG, Bento, RF, Minitti A, Câmara J. Zumbido: Características e Epidemiologia. Experiência do Hospital das Clínicas da Faculdade de Medicina da Universidade de São Paulo. Rev Bras Otorrinolaringol. 1997;63(3):229-35.

27. Jastreboff PJ, Jastreboff MM. First brazilian course on tinnitus retraining therapy for management of tinnitus and hyperacusis [apostilado]. São Paulo: Fundação Otorrinolaringologia; 2001.

28. Tyler R, Coelho C, Tao P, Ji H, Noble W, Gehringer A, et al. Identifying tinnitus subgroups with cluster analysis. Am J Audiol. 2008;17(2):S17684.

29. Mrena R, Ylikoski M, Mäkitie A, Pirvola U, Ylikoski J. Occupational noise-induced hearing loss reports and tinnitus in Finland. Acta Otolaryngol. 2007;127(7):729-35.

30. Babisch W. The noise/stress concept, risk assessment and research needs. Noise Health.2002;4(16):1-11. 